\title{
Accelerometry reveals differences in gait variability between patients with multiple sclerosis and healthy controls
}

\author{
Jessie M. Huisinga ${ }^{1}$, Martina Mancini ${ }^{2}$, Rebecca St. George ${ }^{2}$, and Fay Horak ${ }^{2}$ \\ ${ }^{1}$ University of Kansas Medical Center, Landon Center on Aging, 3901 Rainbow Blvd, Mail stop \\ 1005, Kansas City, KS 66160 \\ 2Oregon Health and Science University, Department of Neurology, NSI Building, 505 NW 185th \\ Ave., Beaverton, OR 97006
}

\begin{abstract}
Variability of movement reflects important information for the maintenance of the health of the system. For pathological populations, changes in variability during gait signal the presence of abnormal motor control strategies. For persons with multiple sclerosis (PwMS), extensive gait problems have been reported including changes in gait variability. While previous studies have focused on footfall variability, the present study used accelerometers on the trunk to measure variability during walking. Thus, the purpose of this study was to examine the variability of the acceleration pattern of the upper and lower trunk in PwMS compared to healthy controls. We extracted linear and nonlinear measures of gait variability from 30s of steady state walking for 15 PwMS and 15 age-matched healthy controls. PwMS had altered variability compared to controls with greater Lyapunov exponent in the ML $(p<0.001)$ and AP $(p<0.001)$ directions, and greater frequency dispersion in the ML direction $(p=0.034)$. PwMS also demonstrated greater mean velocity in the ML direction ( $p=0.045)$ and lower root mean square of acceleration in the AP direction $(p=0.040)$. These findings indicate that PwMS have altered structure of variability of the trunk during gait compared to healthy controls and agree with previous findings related to changes in gait variability in PwMS.
\end{abstract}

\section{Keywords \\ nonlinear; trunk; steady state; Lyapunov exponent}

\section{Introduction}

Multiple sclerosis (MS) is a degenerative neurological disease which affects myelin, oligodendrocytes, and axons ${ }^{40}$ and impacts people early in life. ${ }^{12}$ Due to problems with neural functioning, persons with MS (PwMS) experience both sensory and motor

\footnotetext{
Correspondence should be directed to: Jessie Huisinga, PhD, Landon Center on Aging, 3902 Rainbow Blvd, Mail stop 1005, Kansas City, KS 66160, Phone: (913) 945-7465, Fax: (913) 945-7754, jhuisinga@kumc.edu.

Conflict of Interest

The authors certify that no party having a direct interest in the results of the research supporting this article has or will confer a benefit on us or on any organization with which we are associated.
} 
dysfunction which contributes to problems with balance, coordination, and walking. ${ }^{2,}, 54$ Gait analyses of MS patients reveal abnormalities in speed, stride length, cadence, double support time, swing time, and muscle contraction and relaxation times. ${ }^{2,13}$ Additionally, biomechanical assessment of kinematic gait parameters in PwMS reveals significant changes in lower extremity movement and force production. ${ }^{20,57}$ The causes of such gait dysfunction are complex and incompletely understood. Factors contributing to mobility disorders in MS may include slowed spinal somatosensory conduction ${ }^{4}$, abnormal sensorimotor control ${ }^{10}$, and leg power asymmetry ${ }^{9}$, among others.

Another method to assess changes in gait in PwMS is to evaluate the variability present in the walking pattern since variability reflects important information for the maintenance of the health of the system. ${ }^{14}$ Previous studies have examined gait variability in PwMS and in other neurological disease populations by looking specifically at footfall data and lower extremity movement. Sosnoff et al ${ }^{50}$ reported that PwMS had greater variability in the time between steps, in single support percent, and in step width than controls. Crenshaw ${ }^{11}$ reported that PwMS had significantly greater hip, knee, and ankle joint angle variability than control subjects. Kaipust et $\mathrm{al}^{24}$ found that in PwMS, the stride length and step width time series were more regular and repeatable which the authors interpreted to mean that PwMS have reduced capacity to adapt and respond to perturbations during gait. Because these findings are based solely on footfall and lower extremity data, there is no information provided regarding any specific movement strategies that are employed to control wholebody motion during walking. Since walking is a motor task which requires both forward movement toward a goal and the maintenance of upright posture, evaluating changes in gait variability provides unique information on balance control that is necessary to perform the task. ${ }^{36}$ In the present study, rather than measuring lower extremity movement, we examine the walking pattern in PwMS by investigating the acceleration pattern at the lower (lumbar) and upper (sternum) trunk. Measuring acceleration of the trunk segment during walking is an alternative and suitable method of assessing motion during walking since two-thirds of the body's weight is located at two-thirds of the body's height above the ground..$^{25}$ This technique has been used previously in both healthy ${ }^{32-35}$ and in pathological populations $^{22,23,29}$ to assess movement patterns during walking and has been shown to have good test-retest reliability. ${ }^{17}$ Acceleration of the trunk can also be measured in any environment, laboratory or clinical, with very little equipment which offers an advantage to footfall measures which require some type of footfall detection system. Assessing the acceleration pattern at the lower and upper trunk using two sensors allows for investigation of trunk motion which is responsible for regulating and attenuating gait related oscillations to achieve stabilization for the head's perception of sensorimotor information. ${ }^{55}$ Any alterations in the control of trunk oscillation might be associated with poor balance control during gait. ${ }^{26,36}$ Previously, Sosnoff et al ${ }^{49}$ used accelerometry to examine real-life walking in PwMS by employing both linear and nonlinear measures to assess movement variability. That study evaluated a time series of step counts obtained from an accelerometer worn at the waist over several days, so the construct of the outcome variables in the present study is quite different, but that study does represent a previous application of nonlinear measures applied to accelerometer data in PwMS. The outcome variables described in the present study, both linear and nonlinear variables, have been used extensively in examining the 
center of pressure time series during quiet standing in PwMS ${ }^{19,21,54}$ and in other populations. ${ }^{477,15,18}$ This study, however, represents the application of these outcome variables to assess motion during a single walking task and focused on the motion of the trunk only.

Thus, the purpose of this study was to examine the variability of the movement pattern during walking, as represented by the acceleration time series at the upper and lower trunk, during walking in PwMS. In order to examine variability of trunk motion during gait in PwMS, we used both linear and nonlinear tools to assess both amount and structure of variability within the acceleration pattern. Walking is a continuous movement task which requires dynamic stability, therefore the temporal structure of the movement path can provide information regarding the behavior of the body over time. Linear measures to assess variability provide information on the amount of variability within the signal by employing averaging procedures. These averaging procedures assume that variations between repetitions of a task, in this case consecutive steps, are independent of future and past repetitions. When variability within the time series is measured with nonlinear tools, it helps to explain the adaptive strategies of the system. ${ }^{15,16}$

For this study, we hypothesize that if the stabilogram of acceleration during gait follows that same trend as the center of pressure stabilogram during quiet standing in $\mathrm{PwMS}^{21}$, then we expect PwMS to exhibit a greater range of acceleration and greater root mean square of acceleration at both the sternum and lumbar sensor. In addition, we would expect the acceleration time series to show less divergence (lower LyE) and greater periodicity (lower ApEn) at the lumbar and sternum sensors compared to controls. However, previous studies have described PwMS as having greater variability during gait ${ }^{11,50}$ so if the acceleration time series follows these previous results, then we would expect the acceleration time series to show a greater range of acceleration and greater root mean square of acceleration as well as greater divergence (greater $\mathrm{LyE}$ ) and less periodicity (greater ApEn) of motion at the lumbar and sternum sensor compared to controls.

\section{Materials \& Methods}

\section{Participants}

Patients with MS ( $\mathrm{n}=15)$, recruited through the University's Medical Center, and healthy controls $(n=15)$, recruited through the community, provided informed consent. Patients with MS and healthy controls were matched according to age, height, and mass (Table 1). The research protocol was approved by the University's Institutional Review Board. Inclusion criteria for the MS group was 1) diagnosis of MS made by a neurologist, 2) ability to walk 25 feet without walking aid, 3) no clinical relapses within the previous 60 days, 4) free from any other problems which may affect gait such as vestibular issues, orthopaedic problems, and diabetic neuropathy. Healthy controls were age, height, and mass matched to the MS group and were also free of any conditions which could affect gait. On the day of testing, PwMS completed the self-reported Expanded Disability Status Scale (EDSS). The EDSS is a standard and heavily used disability classification scale for PwMS. ${ }^{28}$ The selfreported EDSS correlates strongly ${ }^{3}$ with the clinician administered version and was utilized 
in this study to avoid requiring MS participants to visit multiple study locations since the laboratory testing location and participating neurologist were located on different campuses.

\section{Walking protocol}

Subjects walked at self-selected pace down a 100 foot hallway, free from any persons, objects, or possible distractors, while wearing 6 MTX Xsens sensors (49A33G15, Xsens, Enschede, NL, USA) sampling at $50 \mathrm{~Hz}$. The sampling frequency was chosen based on examination of the power spectral density of the acceleration signal in pilot subjects which showed the entire signal contained below $5 \mathrm{~Hz}$. The sensors contained 3D accelerometers $( \pm$ $1.7 \mathrm{~g}$ ) and $3 \mathrm{D}$ gyroscopes ( $\pm 300 \%$ s range) mounted on: (i) sternum, (ii) posterior trunk approximately at L5 level (lumbar), (iii) right and left wrist, (iv) right and left lower shank. The sternum and lumber sensors were used to assess trunk motion while the leg sensors were used to assess stride velocity. In order to obtain steady state walking, data analysis was performed on the acceleration time series starting 5 seconds after the onset of the walking trial such that the gait initiation period was not included in the analysis. Only 30 seconds of walking was included for analysis since some subjects took longer than others to complete walking the entire distance.

\section{Data analysis}

The acceleration time series in the medio-lateral (ML) and antero-posterior (AP) directions from the sternum and lumbar sensors were analyzed for 30 seconds of steady state walking. Before extracting the linear variables, 3D acceleration signals from both trunk sensors were transformed to a true horizontal-vertical Cartesian coordinate system ${ }^{38}$ and filtered with a 2 $\mathrm{Hz}$ cut-off, zero-phase, low-pass Butterworth filter. The filtered signal was used to extract the following trunk variables only: range - to quantify the peak-to-peak amplitude of acceleration traces; root mean square acceleration (RMS) - to quantify the dispersion of the acceleration traces; mean velocity - computed by the integration of the acceleration traces; and frequency dispersion - to estimate the variability of the frequency content of the power spectral density of the acceleration traces. Values for frequency dispersion are between 1 and 0 where 0 indicates a purely sinusoidal signal. All variables were calculated using customized MatLab software (The Mathworks Inc., Natick, MA). ${ }^{44}$

Data was analyzed for nonlinear variables unfiltered so as not to mask or remove any dynamical properties or variability present within the system. ${ }^{15}$ Temporal structure of the trunk acceleration time series was quantified from both directions using Lyapunov Exponent (LyE) and approximate entropy (ApEn). The largest LyE is a measure of the rate at which nearby trajectories in state space diverge and the system's sensitivity to initial conditions thereby ${ }^{52}$ Lack of divergence in the acceleration pattern will produce small values for the $\mathrm{LyE}$ and vice versa. The LyE was calculated using customized MatLab software based on Wolf's algorithm ${ }^{56}$ with an embedded dimension of 5 for both groups which was calculated using a Global False Nearest Neighbor analysis. ${ }^{52}$ The time evolution constant was set at 5 for PwMS and at 7 for healthy controls based examination of a range of constants for each group. ApEn quantifies how predictable and regular are data patterns within a time series. ${ }^{42,}{ }^{43}$ ApEn was calculated using customized MatLab software based upon the 
methodology of Pincus ${ }^{42,43}$ (lag $=5, \mathrm{~m}=2, \mathrm{r}=0.2$ were used as default parameters for both groups).

Figure 1A illustrates a stabilogram from both an MS subject (right) and a healthy control (left) created from the acceleration time series. Figure 1B illustrated the ML time series only, taken from the same subject, from which the linear variables were calculated. Figure $1 \mathrm{C}$ is the ML time series plotted against its first derivative to represent the signal in a higher dimension where the presence of an attractor within the time series begins to be visible.

Group means for range, mean velocity, frequency dispersion, RMS, LyE, and ApEn were calculated for healthy controls and PwMS at both the lumbar (sacrum sensor) and sternum. Two separate $2 \times 2$ repeated measures ANOVA models were employed to test for effects of GROUP (MS v. Control) and POSITION (lumbar v. sternum) in the ML and AP directions since directional control of movement is performed independently. ${ }^{48}$ Independent and dependent t-tests were used for post hoc analysis when significant group by condition interactions were identified. Independent t-tests were used to compare demographics between groups. Statistical analysis was performed using SPSS 20.0 (SPSS, Inc., Chicago, IL) with level of significance set at 0.05 .

\section{Results}

The MS group $(75.22 \mathrm{~cm} / \mathrm{s})$ and healthy controls $(76.88 \mathrm{~cm} / \mathrm{s})$ showed no significant differences $(p=0.597)$ in mean stride velocity. Groups were also matched based on age, height, and body mass (Table 1).

\section{Effects of Group}

Linear Measures-In the ML direction, the RMS demonstrated no significant main effect $(p=0.540)$ for Group. Range also demonstrated no significant main effect $(p=0.895)$ for Group but mean velocity did show a significant main effect $(p=0.045)$ where PwMS showed greater values than healthy controls. Frequency dispersion also demonstrated a significant main effect $(p=0.034)$ for Group where PwMS showed values greater than healthy controls.

In the AP direction, RMS demonstrated a significant main effect $(p=0.040)$ for Group where PwMS showed lower values than healthy controls. Range $(p=0.197)$, mean velocity $(p=0.145)$ and frequency dispersion $(p=0.581)$ showed no main effect for Group (Figure 2)

Nonlinear Measures-In the ML direction, LyE demonstrated a significant main effect $(p<0.001)$ for Group where values for PwMS were greater than healthy controls. ApEn demonstrated no effect for Group ( $p=0.264)$.

In the AP direction, LyE demonstrated a significant main effect $(p<0.001)$ for Group were PwMS were greater than healthy. ApEn demonstrated no effect for Group $(p=0.585)$ (Figure 3). 
A main effect for Group was seen in both linear (ML velocity, ML frequency dispersion) and nonlinear (ML \& AP Lyapunov exponent) measures where values for PwMS were significantly greater than in healthy controls.

\section{Effect of Position}

Linear Measures-In the ML direction RMS showed a significant main effect $(p<0.001)$ for Position where the values for RMS were greater in the lumbar position than the sternum. Range also showed a significant main effect $(p<0.001)$ for Position where lumbar was greater than sternum. Velocity also showed a significant main effect $(p=0.001)$ for Position where values for the sternum were greater than lumbar. Frequency dispersion demonstrated a significant main effect $(p<0.001)$ for Position where values were greater at sternum compared to the lumbar sensor.

In the AP direction, the RMS demonstrated a significant main effect $(p<0.01)$ for Position where the lumbar showed greater values than the sternum. Range showed a significant main effect $(p<0.001)$ for Position where lumbar values were greater than the sternum.

Frequency dispersion demonstrated no significant main $(p=0.379)$ for Position. Velocity showed no main effect for Position $(p=0.167)$ (Figure 2).

There were no significant interactions between Group and Position for RMS, range, mean velocity, or frequency dispersion in the ML direction or in the AP direction (Table 2).

Nonlinear Measures-In the ML direction, the LyE showed a significant main effect ( $p$ $<0.001)$ for Position where lumbar values were greater than the sternum. ApEn showed a significant main effect $(p<0.01)$ for Position where lumbar values are greater than sternum.

In the AP direction, the LyE showed no effect for Position $(p=0.095)$. ApEn showed a significant main effect $(p<0.01)$ for Position where sternum values are greater than lumbar (Figure 3).

There were no significant interactions between Group and Position for LyE or ApEn in the ML or the AP direction (Table 2).

A main effect of Position was seen for both linear (ML \& AP root mean square, ML \& AP range, ML velocity, ML frequency dispersion) and nonlinear (ML Lyapunov exponent, ML \& AP approximate entropy) measures.

\section{Discussion}

In this study, we investigated gait variability in PwMS compared to healthy controls by evaluating the acceleration pattern of the trunk during steady state walking. Gait variability was measured using both linear and nonlinear variables in order to describe both the amount and the structure of the variability within the time series. Different gait variability measures represent different constructs and should be included in gait analysis to enhance our understanding of variability in gait. ${ }^{37}$ Our findings indicate that acceleration of the trunk during walking in PwMS had a larger frequency dispersion in the ML direction, but in the AP direction, RMS was lower in PwMS compared to controls. The acceleration of the trunk 
in PwMS also exhibits greater divergence (LyE) in both the ML and AP directions. Mean velocity was greater in PwMS in the ML direction only. These findings generally agree with previous studies which have reported increased amount of gait variability in PwMS. ${ }^{11,50}$ We also found several differences in variability of trunk acceleration due to position of the sensor, but no interactions between group and position effects which indicates that we did not identify differences in movement strategy of the lower vs. upper segments of the trunk between the groups.

It is important to note that there were no statistical differences in stride velocity between the groups (Table 1) and therefore both groups walked at effectively the same speed. Large differences in walking speed could imply that increases in trunk acceleration could be attributed to an unsteady speed due to pathology. Because the groups walked at effectively the same speed, we did not need to normalize the outcome variables to walking speed. ${ }^{22}$

Frequency dispersion effectively quantifies the sinusoidal properties of the power spectrum of the acceleration signal where values closer to zero are indicative of a purely sinusoidal signal. ${ }^{8}$ PwMS exhibited greater frequency dispersion than controls in the ML direction which indicates that the acceleration signal was less periodic with more frequencies included in the signal indicating greater variability of the acceleration frequencies. This finding, along with increased LyE for PwMS, indicates an acceleration pattern of the trunk during gait that is less periodic and contains greater divergence in the ML direction compared to healthy controls. It is possible that PwMS control for greater divergence of the trunk in the ML direction by increasing step width ${ }^{45}$ as control of motion in the ML is thought to require more step-by-step control compared to the AP direction. ${ }^{27,41}$ The role of lateral trunk motion is a key component of dynamic balance control during gait. ${ }^{1,27}$ Accordingly, loss of sensory information could impact the active adjustment required for lateral control. ${ }^{27}$ PwMS have previously demonstrated decrements in postural control due to changes in sensory input $^{6}$, but during gait, the impact of sensory loss has not been quantified. While this study did not specifically remove sensory information during gait, PwMS have demonstrated loss of somatosensory information during standing tasks ${ }^{4}$. Thus, it is likely that altered somatosensation in PwMS could likely impact gait. In addition to loss of sensory input, up to $80 \%$ of PwMS report problems with spasticity, the velocity-dependent increased tonic stretch reflexes and exaggerated tendon jerks resulting from hyper-excitability of the stretch reflex. ${ }^{30,46}$ Problems with spasticity could account for the increased ML velocity of the lower and upper trunk in PwMS compared to healthy controls found in the present study, though spasticity is typically reported in the lower extremities. ${ }^{46} \mathrm{We}$ did not directly measure spasticity for the included subjects, but it would be of interest to examine the relationship between spasticity reports and control of the upper and lower trunk during walking.

In the AP direction, the results do not strictly identify an increase in variability during gait. RMS was actually decreased in PwMS which would indicate that the amount of variability was lower. If we consider motion in the AP direction to exhibit more passive dynamics ${ }^{41}$, then lower RMS for PwMS could indicate less overall peak-to-peak motion of the trunk in the AP direction during gait. Spain et $\mathrm{al}^{51}$ actually reported increased trunk motion in PwMS, but only in the lateral direction. Also in the AP direction, LyE was increased in 
PwMS which indicates greater divergence of the movement pattern. LyE quantifies separation between continuous paths of movement and whether these paths will expand or contract within a dynamical system. ${ }^{52}$ Increased LyE values for PwMS in both the ML and AP directions compared to controls indicate an acceleration pattern with more divergence and reduced complexity. The change in complexity in the PwMS corresponds well with the optimal movement variability model. This theory states that optimal movement variability has a highly complex structure which is associated with healthy movement patterns and reflects a rich behavioral state that allows for diverse movement strategies. Systems that are either too variable or too periodic reflect reduced complexity. ${ }^{53}$ Effectively, variability reflects multiple options for movement, providing for flexible, adaptive strategies that are not reliant on specified programs for each task or for each changing condition encountered. ${ }^{14}$ Too much variability, such as in individuals with an ataxic movement disorder which is characteristic for $\mathrm{PwMS}^{51}$, can be a problem and indicates a less complex system which has excessive degrees of freedom. ${ }^{39}$ For example, if movement during a healthy individuals gait typically falls within a certain range of variability, then increasing the variability beyond this range will lead to disturbances in the movement pattern. In other words, if the variability is too high, then unexpected disturbances in one gait cycle will impact the next gait cycle and so on. Gait is an example of a cyclic task which is continuous and cannot be too variable else an individual will not be able maintain control from one cycle to the next. Alternatively, the task cannot be too periodic else the pattern would be completely unadaptable. ${ }^{14}$ It appears that compared to controls, PwMS are effectively too variable in their trunk movement pattern during gait. Too much variability in the movement pattern could help explain the high incidence of falls reported by $\mathrm{PwMS}^{5}$ and agrees with the Lipsitz and Goldberg hypothesis of loss of complexity of a physiological or behavioral control system with age and disease. ${ }^{31}$ It is not yet clear what relationship gait variability has with other clinical assessment measures in PwMS, thus specific suggestions regarding the clinical interpretation of the present study's findings may be premature. From a clinical perspective, it may be of interest to examine the relationship between excessive gait variability and increased falls in PwMS.

This study also found several differences in variability of the lower trunk (lumbar) compared to the upper trunk (sternum). Overall, these findings indicate greater variability at the lumbar compared to the sternum. This finding fits well with the goal of attenuation of movement of the head where motion of the upper trunk compared to the lower trunk would be limited in order to stabilize the head. ${ }^{55}$ Because there were no interactions between Group and Position, it appears that both groups demonstrate the same strategy in terms of dampening motion of the trunk from the lower to upper position. These findings may indicate that positioning the sensor at the lumbar position, rather than the sternum, to study variability of trunk motion during gait will provide more relevant information.

Employing different measures of variability to examine the acceleration signal allowed us to gain a unique perspective on control during gait in PwMS. The use of accelerometers also allowed us to examine continuous gait outside of the laboratory and without the use of a treadmill. In PwMS, the acceleration time series showed increased frequency dispersion in the ML direction and increased LyE in both the ML and AP direction which indicates excessive divergence and reduced behavioral complexity as compared to controls. One 
limitation of this study is only PwMS who were independent ambulators and did not require a walking aid were evaluated. This makes our results generalizable only to PwMS who don't use a walking aid though it would be of interest to perform this evaluation on a wider range of disability levels. Future studies should also investigate the relationship between gait and specific system (sensory, pyramidal, cerebellar) disability to determine whether disability of a specific subsystem is related to changes in gait variability. In addition, direct measurement of altered somatosensation and the relationship with gait could identify whether a common sensory input impacts changes in gait variability.

\section{Supplementary Material}

Refer to Web version on PubMed Central for supplementary material.

\section{Acknowledgments}

This study was funded by the National Multiple Sclerosis Society's Rehabilitation Research Training Grant and the Medical Research Foundation of Oregon's Early Clinical Investigator Award.

\section{References}

1. Bauby CE, Kuo AD. Active control of lateral balance in human walking. Journal of Biomechanics. 2000; 33:1433. [PubMed: 10940402]

2. Benedetti MG, Piperno R, Simoncini L, Bonato P, Tonini A, Giannini S. Gait abnormalities in minimally impaired multiple sclerosis patients. Multiple sclerosis (Houndmills, Basingstoke, England). 1999; 5:363.

3. Bowen J, Gibbons L, Gianas A, Kraft GH. Self-administered Expanded Disability Status Scale with functional system scores correlates well with a physician-administered test. Mult Scler. 2001; 7:201-206. [PubMed: 11475445]

4. Cameron MH, Horak FB, Herndon RR, Bourdette D. Imbalance in multiple sclerosis: a result of slowed spinal somatosensory conduction. Somatosensory \& motor research. 2008; 25:113. [PubMed: 18570015]

5. Cattaneo D, De Nuzzo C, Fascia T, Macalli M, Pisoni I, Cardini R. Risks of falls in subjects with multiple sclerosis. Archives of Physical Medicine and Rehabilitation. 2002; 83:864. [PubMed: 12048669]

6. Cattaneo D, Jonsdottir J. Sensory impairments in quiet standing in subjects with multiple sclerosis. Multiple sclerosis (Houndmills, Basingstoke, England). 2009; 15:59.

7. Cavanaugh JT, Guskiewicz KM, Giuliani C, Marshall S, Mercer VS, Stergiou N. Recovery of postural control after cerebral concussion: new insights using approximate entropy. Journal of athletic training. 2006; 41:305. [PubMed: 17043699]

8. Chiari L, Rocchi L, Cappello A. Stabilometric parameters are affected by anthropometry and foot placement. Clinical biomechanics (Bristol, Avon). 2002; 17:666.

9. Chung LH, Remelius JG, Van Emmerik RE, Kent-Braun JA. Leg power asymmetry and postural control in women with multiple sclerosis. Medicine and science in sports and exercise. 2008; 40:1717. [PubMed: 18799980]

10. Cowan JM, Rothwell JC, Dick JP, Thompson PD, Day BL, Marsden CD. Abnormalities in central motor pathway conduction in multiple sclerosis. Lancet. 1984; 2:304. [PubMed: 6146860]

11. Crenshaw SJ, Royer TD, Richards JG, Hudson DJ. Gait variability in people with multiple sclerosis. Multiple sclerosis (Houndmills, Basingstoke, England). 2006; 12:613.

12. Frohman EM. Multiple sclerosis. The Medical clinics of North America. 2003; 87:867. [PubMed: 12834152] 
13. Gehlsen G, Beekman K, Assmann N, Winant D, Seidle M, Carter A. Gait characteristics in multiple sclerosis: progressive changes and effects of exercise on parameters. Archives of Physical Medicine and Rehabilitation. 1986; 67:536. [PubMed: 3741079]

14. Harbourne RT, Stergiou N. Movement variability and the use of nonlinear tools: principles to guide physical therapist practice. Physical Therapy. 2009; 89:267. [PubMed: 19168711]

15. Harbourne RT, Stergiou N. Nonlinear analysis of the development of sitting postural control. Developmental psychobiology. 2003; 42:368. [PubMed: 12672087]

16. Hausdorff JM, Purdon PL, Peng CK, Ladin Z, Wei JY, Goldberger AL. Fractal dynamics of human gait: stability of long-range correlations in stride interval fluctuations. Journal of applied physiology (Bethesda, Md: 1985). 1996; 80:1448.

17. Henriksen M, Lund H, Moe-Nilssen R, Bliddal H, Danneskiod-Samsoe B. Test-retest reliability of trunk accelerometric gait analysis. Gait \& posture. 2004; 19:288-297. [PubMed: 15125918]

18. Hong SL, Manor B, Li L. Stance and sensory feedback influence on postural dynamics. Neuroscience letters. 2007; 423:104. [PubMed: 17673367]

19. Huisinga JM, Filipi M, Stergiou N. Supervised resistance training results in changes in postural control in multiple sclerosis patients. Motor control. 2011; 16:50-63. [PubMed: 22402220]

20. Huisinga JM, Schmid KK, Filipi ML, Stergiou N. Persons With Multiple Sclerosis Show Altered Joint Kinetics During Walking After Participating in Elliptical Exercise. J Appl Biomech. 2012; 28:249-257. [PubMed: 21975419]

21. Huisinga JM, Yentes JM, Filipi ML, Stergiou N. Postural control strategy during standing is altered in patients with multiple sclerosis. Neurosci Lett. 2012; 524:124-128. [PubMed: 22824302]

22. Iosa M, Fusco A, Morone G, Pratesi L, Coiro P, Venturiero V, De Angelis D, Bragoni M, Paolucci S. Assessment of upper-body dynamic stability during walking in patients with subacute stroke. $\mathrm{J}$ Rehabil Res Dev. 2012; 49:439-450. [PubMed: 22773202]

23. Iosa M, Mazza C, Pecoraro F, Aprile I, Ricci E, Cappozzo A. Control of the upper body movements during level walking in patients with facioscapulohumeral dystrophy. Gait \& posture. 2010; 31:68-72. [PubMed: 19782569]

24. Kaipust JP, Huisinga JM, Filipi M, Stergiou N. Gait variability measures reveal differences between multiple sclerosis patients and healthy controls. Motor Control. 2012; 16:229-244. [PubMed: 22615327]

25. Kavanagh JJ, Barrett RS, Morrison S. Upper body accelerations during walking in healthy young and elderly men. Gait \& posture. 2004; 20:291-298. [PubMed: 15531176]

26. Kavanagh JJ, Menz HB. Accelerometry: a technique for quantifying movement patterns during walking. Gait \& posture. 2008; 28:1-15. [PubMed: 18178436]

27. Kuo A. Stabilization of lateral motion in passive dynamic walking. International Journal of Robot Research. 1999; 18:917-930.

28. Kurtzke JF. Rating neurologic impairment in multiple sclerosis: an expanded disability status scale (EDSS). Neurology. 1983; 33:1444. [PubMed: 6685237]

29. Lamoth CJ, Meijer OG, Wuisman PI, van Dieen JH, Levin MF, Beek PJ. Pelvis-thorax coordination in the transverse plane during walking in persons with nonspecific low back pain. Spine. 2002; 27:E92-99. [PubMed: 11840116]

30. Lance JW. The control of muscle tone, reflexes, and movement: Robert Wartenberg Lecture. Neurology. 1980; 30:1303-1313. [PubMed: 7192811]

31. Lipsitz LA, Goldberger AL. Loss of 'complexity' and aging. Potential applications of fractals and chaos theory to senescence. JAMA : the journal of the American Medical Association. 1992; 267:1806-1809. [PubMed: 1482430]

32. Marigold DS, Patla AE. Age-related changes in gait for multi-surface terrain. Gait $\&$ posture. 2008; 27:689-696. [PubMed: 17962018]

33. Mazza C, Iosa M, Pecoraro F, Cappozzo A. Control of the upper body accelerations in young and elderly women during level walking. Journal of neuroengineering and rehabilitation. 2008; 5:30. [PubMed: 19014631]

34. Mazza C, Iosa M, Picerno P, Cappozzo A. Gender differences in the control of the upper body accelerations during level walking. Gait \& posture. 2009; 29:300-303. [PubMed: 19013799] 
35. Mazza C, Zok M, Cappozzo A. Head stabilization in children of both genders during level walking. Gait \& posture. 2010; 31:429-432. [PubMed: 20163963]

36. Moe-Nilssen R. A new method for evaluating motor control in gait under real-life environmental conditions. Part 2: Gait analysis. Clin Biomech (Bristol, Avon). 1998; 13:328-335.

37. Moe-Nilssen R, Aaslund MK, Hodt-Billington C, Helbostad JL. Gait variability measures may represent different constructs. Gait \& posture. 2010; 32:98-101. [PubMed: 20434916]

38. Moe-Nilssen R, Helbostad JL. Trunk accelerometry as a measure of balance control during quiet standing. Gait \& posture. 2002; 16:60-68. [PubMed: 12127188]

39. Newell, KM. Applications of nonlinear dynamics to developmental process modeling. In: Newell, KM.; Molenaar, PMC., editors. Degrees of freedom and the development of center of pressure profiles. Erlbaum; Hillsdale, NJ: 1997. p. 63

40. Noseworthy JH, Lucchinetti C, Rodriguez M, Weinshenker BG. Multiple sclerosis. The New England journal of medicine. 2000; 343:938. [PubMed: 11006371]

41. O'Connor SM, Kuo AD. Direction-dependent control of balance during walking and standing. Journal of neurophysiology. 2009; 102:1411. [PubMed: 19553493]

42. Pincus SM. Approximate entropy as a measure of system complexity. Proceedings of the National Academy of Science U S A. 1991; 88:2297.

43. Pincus SM I, Gladstone M, Ehrenkranz RA. A regularity statistic for medical data analysis. Journal of clinical monitoring. 1991; 7:335. [PubMed: 1744678]

44. Prieto TE, Myklebust JB, Hoffmann RG, Lovett EG, Myklebust BM. Measures of postural steadiness: differences between healthy young and elderly adults. IEEE transactions on biomedical engineering. 1996; 43:956. [PubMed: 9214811]

45. Remelius JG, Jones SL, House JD, Busa MA, Averill JL, Sugumaran K, Kent-Braun JA, Van Emmerik RE. Gait impairments in persons with multiple sclerosis across preferred and fixed walking speeds. Arch Phys Med Rehabil. 2012; 93:1637-1642. [PubMed: 22559932]

46. Rizzo MA, Hadjimichael OC, Preiningerova J, Vollmer TL. Prevalence and treatment of spasticity reported by multiple sclerosis patients. Mult Scler. 2004; 10:589-595. [PubMed: 15471378]

47. Rocchi L, Chiari L, Horak FB. Effects of deep brain stimulation and levodopa on postural sway in Parkinson's disease. Journal of neurology, neurosurgery, and psychiatry. 2002; 73:267.

48. Shumway-Cook, A.; Woollacott, MH. Motor Control: Theory and Practical Applications. Lippincott Williams \& Wilkins; New York, NY: 2001.

49. Sosnoff JJ, Goldman MD, Motl RW. Real-life walking impairment in multiple sclerosis: preliminary comparison of four methods for processing accelerometry data. Mult Scler. 2010; 16:868-877. [PubMed: 20534642]

50. Sosnoff JJ, Sandroff BM, Motl RW. Quantifying gait abnormalities in persons with multiple sclerosis with minimal disability. Gait \& posture. 2012

51. Spain RI, St George RJ, Salarian A, Mancini M, Wagner JM, Horak FB, Bourdette D. Body-worn motion sensors detect balance and gait deficits in people with multiple sclerosis who have normal walking speed. Gait \& posture. 2012

52. Stergiou, N.; Buzzi, UH.; Kurz, MJ.; Heidel, J. Nonlinear tools in human movement. In: Stergiou, N., editor. Innovative analyses for human movement. Human Kinetics; Champaign, IL: 2004. p. 63-90.

53. Stergiou N, Harbourne R, Cavanaugh J. Optimal movement variability: a new theoretical perspective for neurologic physical therapy. Journal of neurologic physical therapy : JNPT. 2006; 30:120. [PubMed: 17029655]

54. Van Emmerik RE, Remelius JG, Johnson MB, Chung LH, Kent-Braun JA. Postural control in women with multiple sclerosis: effects of task, vision and symptomatic fatigue. Gait \& posture. 2010; 32:608. [PubMed: 20943393]

55. Wilhelmsen K, Nordahl SH, Moe-Nilssen R. Attenuation of trunk acceleration during walking in patients with unilateral vestibular deficiencies. Journal of vestibular research : equilibrium \& orientation. 2010; 20:439-446. [PubMed: 21248407]

56. Wolf A, Swift JB, Swinney HL, Vastano JA. Determining Lyapunov exponents from a time series. Physica D. 1985; 16:285-317. 
57. Wurdeman SR, Huisinga JM, Filipi M, Stergiou N. Multiple sclerosis affects the frequency content in the vertical ground reaction forces during walking. Clin Biomech (Bristol, Avon). 2011; 26:207-212. 

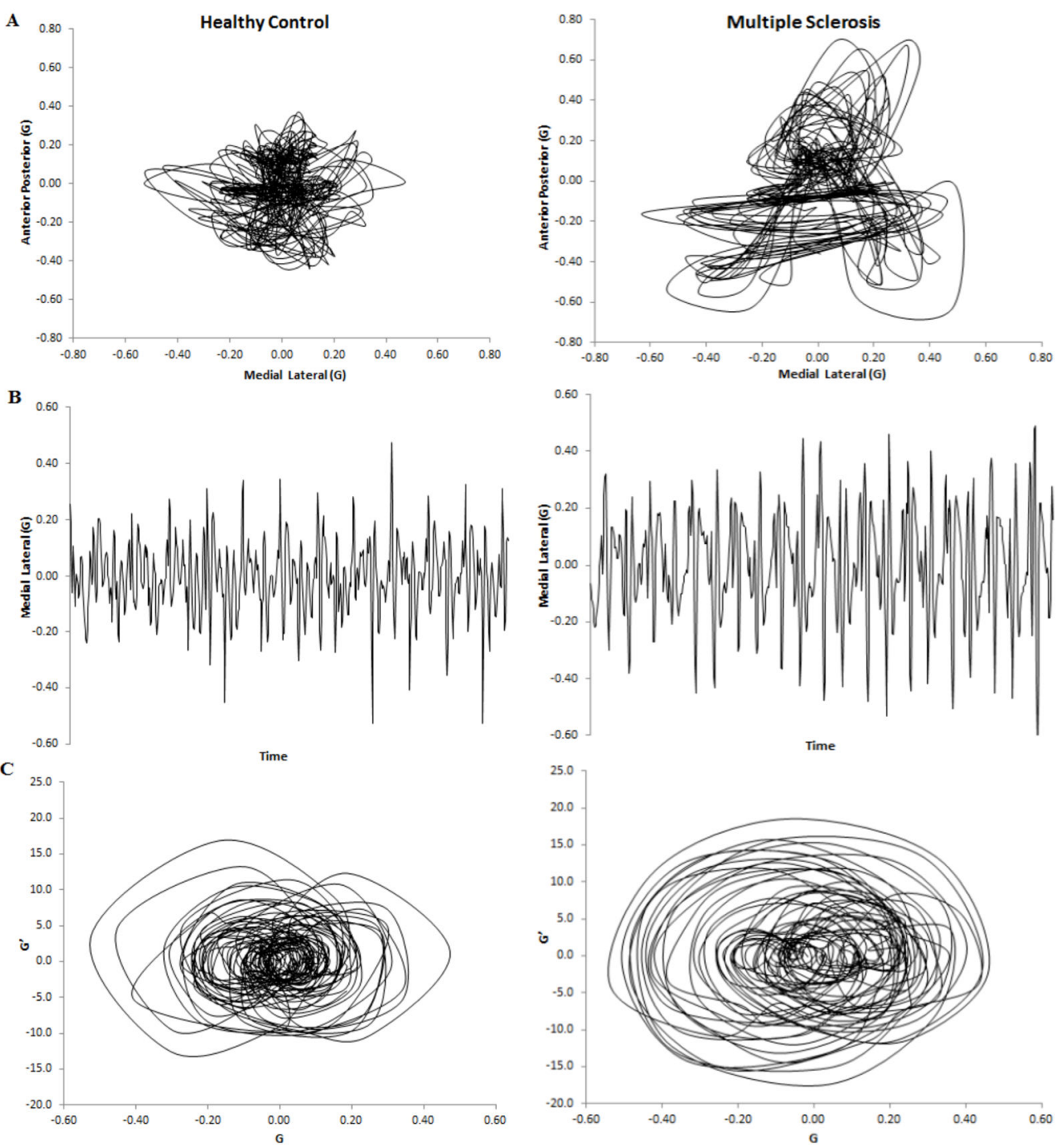

Figure 1.

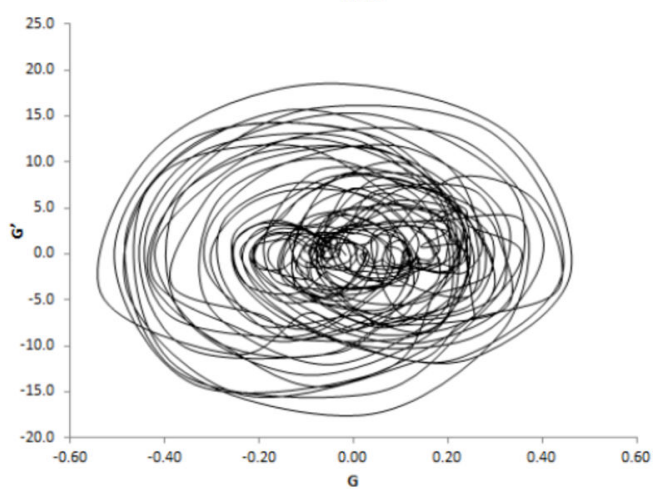



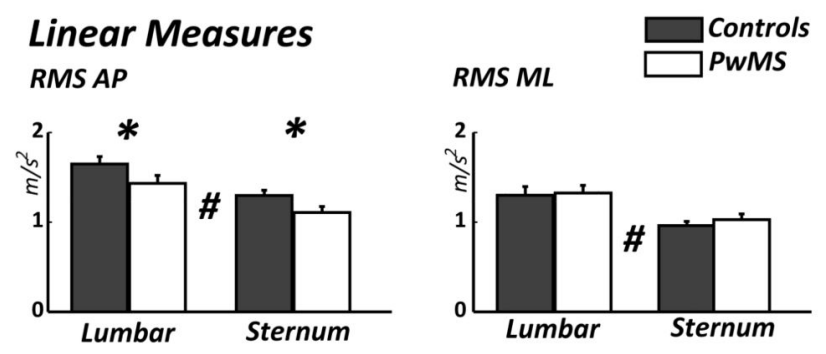
Range AP Range $M L$
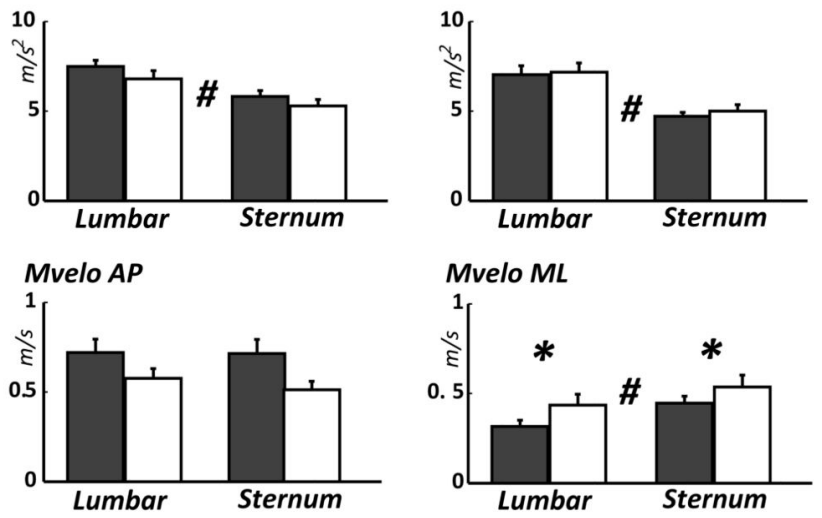

Mvelo ML
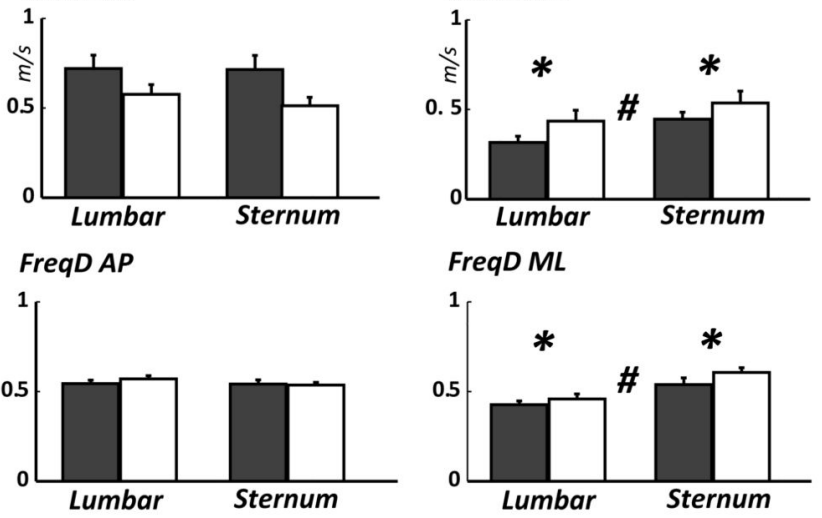
FreqD $M L$

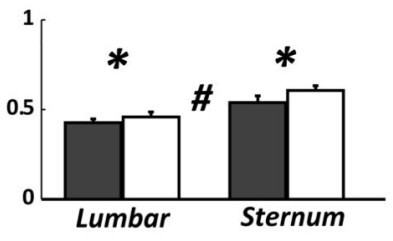

Figure 2. 


\section{Nonlinear Measures}

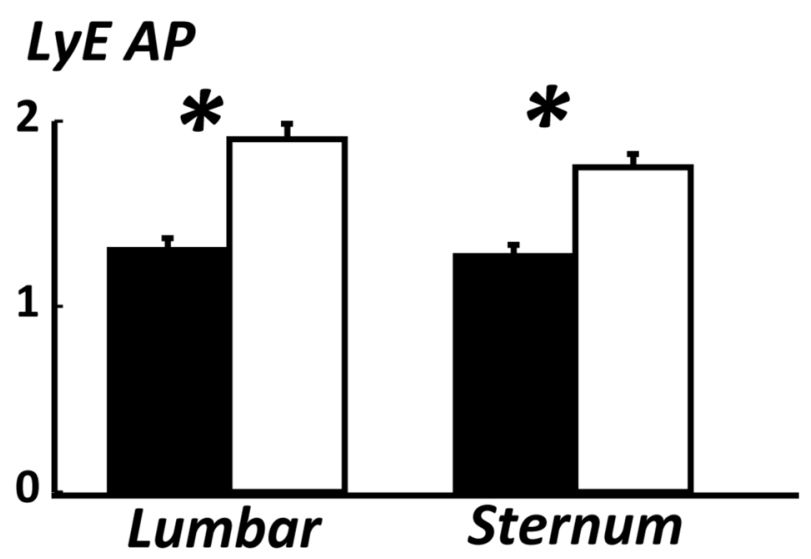

\section{LyE ML}

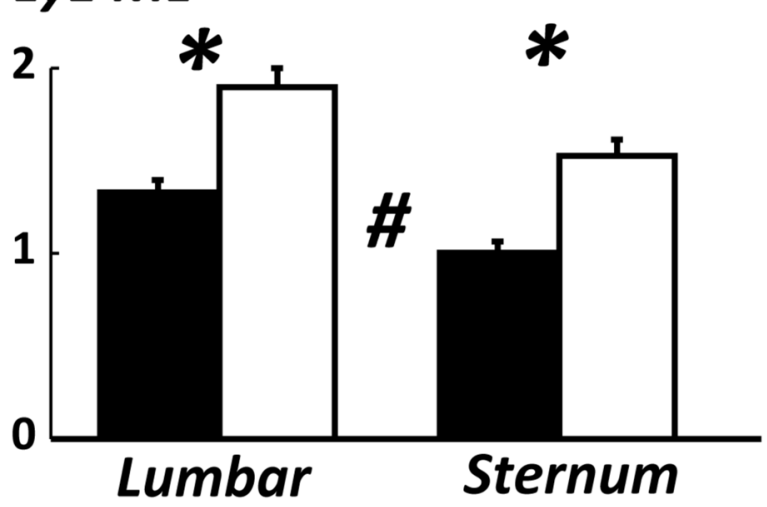

ApEn AP

ApEn ML
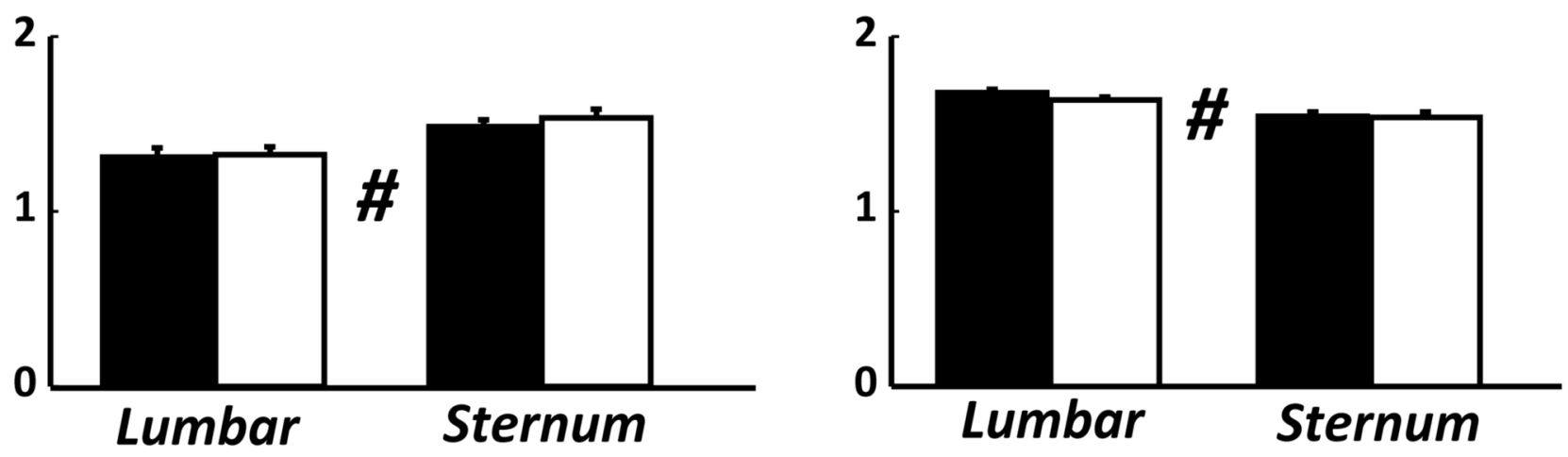

Figure 3. 


\section{Table 1}

Demographics of PwMS and healthy control participants.

\begin{tabular}{lccc}
\hline & MS (n=15) & Control $(\mathbf{n = 1 5})$ & p-value \\
\hline Age $(\mathrm{yrs})$ & $43.75 \pm 11.9$ & $42.20 \pm 10.31$ & 0.703 \\
Height $(\mathrm{cm})$ & $168.96 \pm 9.43$ & $166.13 \pm 18.13$ & 0.598 \\
Mass $(\mathrm{kg})$ & $71.19 \pm 15.69$ & $71.07 \pm 20.06$ & 0.985 \\
Gender & $11 \mathrm{~F} / 4 \mathrm{M}$ & $12 \mathrm{~F} / 3 \mathrm{M}$ & - \\
Stride velocity $(\mathrm{cm} / \mathrm{s})$ & $75.22 \pm 8.35$ & $76.88 \pm 8.64$ & 0.597 \\
Self-reported EDSS mean (IQR) & $4.21(1.0)$ & - & - \\
\hline
\end{tabular}

EDSS - Expanded Disability Status Scale. 
